L. Goldberg: The model can easily be modified in this direction. Contrary to my earlier belief, the most important factor in the widening of the profile at the limb is the limb darkening in the continuum rather than the $\sec \theta$ factor.

H. ALFVEN: Which gas density has been assumed?

L. GolDBERG: It is not necessary at this time to make assumptions as to the gas density, since only radiation damping is required to account for the wing broadening. The theory gives only the total number of two-quantum atoms in the line of sight and the values obtained agree closely with those found by Mustel and Severny.

\title{
6. FLARE-ASSOCIATED SOLAR PHENOMENA
}

\section{BY W. O. Roberts}

Flares are the most abrupt, and in many ways the most spectacular, of solar phenomena. Thus, it is not surprising that associated with flares are a number of other optically observed effects in the solar corona, prominences and plages.

As Dr Ellison states and Dr McMath's superb films show, flares almost always arise from plages(r) and they are most likely to occur in unusually bright or fluctuating plages associated with complex or bipolar sunspot groups, particularly in the first few days of development of spot groups when the plages are usually luminous. Sometimes the occurrence of a flare leaves the plage brighter and larger than it was before the flare (2). Strangely enough, flares rarely show any associated photospheric effects. A few years ago some workers felt that there was an area reduction in sunspots immediately after the outbreak of flares, but Newton and Howe have now shown that there appears to be no such area discontinuity, either in umbra or penumbra, at the time of flares (3).

Flares are generally accompanied by high-speed surge prominences that are sometimes of spectacular behaviour (4). They are visible in hydrogen as 'high-speed dark filaments' on the solar disk, or at the limb as surge prominences. Many of these rise to heights as great as $100,000 \mathrm{~km}$. and some achieve velocities of over $500 \mathrm{~km}$./s. Typical flare surges appear at the time of maximum intensity of the flare, and are already rapidly moving when first observed. Often they decelerate under the influence of forces that exceed solar gravity, finally coming to rest, and then returning to the photosphere along the trajectory they had followed in their outwards course. It is widely speculated that surge prominences are clouds of hydrogen gas set into motion by absorption of the powerful blast of $\mathrm{L} \alpha$ radiation that results from the main 'flash' of the flare maximum. The initial point at which such surges appear is generally near the flare, but not directly over it.

Dr Bruzek has done so much important work on the effects of flares on other solar phenomena that I feel it should be he rather than I who is giving this paper. He has, for example, shown(5) from the observations made at Kanzelhöhe that dark hydrogen filaments, seen in projection on the solar disk, often feel the effects of solar flares, even when they are at distances as great as several hundred thousand kilometres from the flares. Such filaments can be 'activated' by some invisible disturbance that appears to propagate from the flare with velocities from about 20 to about $200 \mathrm{~km}$./ $/ \mathrm{s}$. When activated, the filaments become darker and larger, display sudden changes of shape and position and develop line-of-sight velocities. Sometimes, in cases of nearby dark filaments, a travelling disturbance can be detected propagating along the length of the filament. In other cases, the activated filament may lie at a substantial distance, and may possess no obvious association with the spot group in which the flare has occurred, so that its activation takes place some time later, and might, therefore, easily be thought an independent event. In some cases the flare-activation of a dark filament may lead to its destruction. On the other hand, nearby dark filaments sometimes exhibit little or no response to flares. 
Bruzek has also described an interesting relationship between flare-like brightenings in undisturbed solar areas and sudden eruptions of quiescent dark filaments (disparitions brusques) (6). In this case, the flare-like brightening follows the filament eruption. Most often the consequence of the eruption is a sudden brightening to plage intensity in a nearby little-disturbed chromospheric region. Occasionally, however, the brightenings are sufficient to be classed as ordinary flares. This phenomenon has a most provocative relationship to the corona, and to the outbreak of new sunspot groups. Bruzek has shown, in the same paper, that when a dark filament suddenly erupts it is likely to do so in apparent response to the arrival of a disturbance propagated with the slow velocity of about $\mathrm{I} \mathrm{km}$./s. from a newly formed sunspot group. He has suggested( $)$ that this slowly propagated disturbance is closely associated with the expanding strong-emission contours of the emission-line corona seen in $\lambda 5303$ and with the spreading area of the associated $\mathrm{Ca}$ and $\mathrm{H}$ plages, both of which move out from new sunspot regions with speeds of the order of 0.3 to $0.6 \mathrm{~km}$. $/ \mathrm{s}$. On the other hand, we have been inclined to consider the expanding coronal contours a consequence of the events associated with the heightened flare activity associated with the first few days of development of a spot group (8).

The intensity of radiation from the higher ionization lines of the emission-line corona shows strong enhancement at the times of flares. The yellow coronal line, $\lambda 5694$ for example, shows its strongest emission in clear association with flares(9). This line has recently been shown conclusively to have its origin in $\mathrm{Ca} \mathrm{XV(xo),} \mathrm{as} \mathrm{Edlén} \mathrm{and} \mathrm{Waldmeier}$ had previously indicated, but others had disputed. The identification means that this is the highest ionization line yet found in the corona, with an ionization potential of $8 \mathrm{I} 4 \mathrm{eV}$. This argues for extremely high temperatures in the coronal neighbourhood of flares, a fact also confirmed by coronal line-profile studies in regions near flares(xi).

So far as Climax observations go, the few clear-cut pronounced cases of asymmetry in coronal line-profiles, which presumably result from Doppler displacement of the emission of moving coronal clouds, are found in the close vicinity of limb flares. Moreover, coronal emissions often show sharp concentrations and very high intensities near such regions. The famous west limb region of I9 November I949 is an outstanding example of these phenomena.

Thus, it seems very certain that heightened coronal emission intensities develop after the outbreak of new active regions, and perhaps specifically after the onset of the heightened flare activity of a new region. It also seems quite certain that the increased coronal emission associated with a flare decays appreciably more slowly than the flare emissions themselves. Petri has suggested(I2), moreover, that sharp rises of coronal emission often occur, but that equally sharp declines are absent. This further suggests the origin of strongly heated coronal centres in events with the time-scale of flares.

It would be incorrect, however, to assume that strong coronal emission regions are uniquely associated with flares. Strong coronal regions are associated with centres of activity of the Sun, but sometimes centres with relatively few flares develop very strong coronal emission line-intensities, as in the outstanding new spot-cycle coronal region that passed the east solar limb at $35^{\circ} \mathrm{N}$. latitude on 2 October I954. As in this instance, however, it is difficult to be sure that a pronounced outbreak of flares did not take place on the invisible solar disk. Probably the density of coronal gases is primarily controlled by active region phenomena other than flares, and the basic heat supply for the corona is not associated with flares. But it is also likely that flares or the events that cause them can also be responsible for abrupt and strong elevations of the temperatures of associated coronal regions.

I wish to mention, now, rather parenthetically, a new observation about a few outstanding flare regions that we have made this summer at H.A.O. through the efforts of Dr Malcolm Corell and his assistants Miss Martha Hazen and $\mathrm{Mr}$ John Bahng. An outstanding flare region passed the east solar limb at about $25^{\circ} \mathrm{N}$. on 26 February I947. Following up a suggestion made a number of years ago by Dr Helen Dodson, we traced all possible prominence trajectories on $\mathrm{H} \alpha$ cinematographic films for the limb presentations of this region-going several days from limb passage. The trajectories of the prominence motions show a surprisingly good fit to a dipole magnetic 
field placed with its axis approximately normal to the solar surface, but submerged by 0.2 solar radii below the photosphere. If these trajectories define the true solar atmospheric magnetic field above the chromosphere, as I suspect they do, then the sunspot fields and thus the flare hydromagnetics present an intriguing and difficult problem to interpret.

At present, observations of several sorts are of great importance. First, it is most important to have rapid sequence studies of photospheric magnetic fields in the vicinity of flares, using appropriate spectrum lines, and techniques similar to those of Babcock (13). Secondly, it is very important to have especially complete observations of prominence and coronal phenomena associated with limb flares, so that flare-heights, flare line-widths, velocities of prominences and other characteristics can be related to the behaviour of the corona lines at the same time. Special rapid-sequence coronal studies should be performed with suitable instruments at such times. Efforts should also be made to obtain helium and hydrogen line-spectra, at the limb, with adequate spectroscopic resolution, suitable for derivation of temperature and density estimates for the flare-associated chromospheric plages and prominences.

\section{REFERENCES}

(I) Ellison, Publ. Roy. Obs. Ediṇburgh, vol. I, no. 5, p. 75, 1952.

(2) Krisenko, Bull. Comm. for the Investigation of the Sun, no. 8-9, p. I I, I954, and other authors.

(3) Newton and Howe, Observatory, 72, I I I, I952.

(4) Giovanelli, $A p . J$. 91, 334, 1940; Ellison, M.N. ro9, 3, 1949; Dodson and McMath, $A p . J$. 115, 78, 1952; Bartlett, Witte and Roberts, $A p . J$. 117, 292, 1953.

(5) Bruzek, Z.Ap. 31, 99, I952.

(6) Bruzek, $Z$. Ap. 31, III, 1952; Observatory, 72, 154, 1952.

(7) Bruzek, Z. Ap. 35, 2 1 3, I955.

(8) Billings, Liebenberg and Roberts, Astron. J. 58, 2 I I, 1953.

(9) Waldmeier, Astr. Mitt. Eidgenöss. Sternwarte. Sternwarte Zurich, no. 146, p. 6, 1945.

(10) Pecker, Billings and Roberts, Ap. J. 120, 509, 1954; Layzer, M.N. (in the Press).

(II) Billings, Pecker and Roberts, C.R. 238, I194, 1954; Billings, Hirsch and Varsavsky, Ap. J. x23, 532, 1956 .

(12) Petri, Z. Ap. 34, 68, 1954 .

(13) Babcock, Ap.J. x18, 387, 1953.

\section{DISCUSSION}

M. J. W. DUnGEY: Some lines in your diagram of the ideal dipole cross each other. How can this be?

W. O. RoBERTS: The lines shown included a number projected on the plane of the screen even though these referred to lines in other planes.

T. G. CowlING: Is the enhanced coronal activity associated with flares purely optical, or is there an enhancement of motions?

W. O. RoBERTS: Such evidences are quite rare. The few Doppler displacements known from Climax data are all associated with flare active regions, but correspond to very modest velocities of the order of $25 \mathrm{~km} . / \mathrm{s}$. very near the centre. 